\title{
The clinicopathological and MRI features of patients with BRCA1/2 mutations in familial breast cancer
}

\author{
Chao You ${ }^{1 "}$, Qin Xiao" ${ }^{1 \#}$, Xinyi Zhu ${ }^{2}$, Yiqun Sun ${ }^{1}$, Genhong Di $^{2}$, Guangyu Liu ${ }^{2}$, Yifeng Hou ${ }^{2}$, \\ Canming Chen ${ }^{2}$, Jiong $\mathrm{Wu}^{2}$, Zhimin Shao ${ }^{2}$, Yajia Gu${ }^{1}$, Zhen $\mathrm{Hu}^{2}$ \\ ${ }^{1}$ Department of Radiology, Fudan University Cancer Center, Department of Oncology, Shanghai Medical College, Fudan University, Shanghai, \\ China; ${ }^{2}$ Department of Breast Surgery, Key Laboratory of Breast Cancer in Shanghai, Fudan University Shanghai Cancer Center, Department of \\ Oncology, Shanghai Medical College, Fudan University, Shanghai, China \\ Contributions: (I) Conception and design: C You, Q Xiao, X Zhu; (II) Administrative support: Z Hu, C You; (III) Provision of study materials or \\ patients: C You, Q Xiao; (IV) Collection and assembly of data: C You, Q Xiao, X Zhu, Y Sun; (V) Data analysis and interpretation: C You, Z Hu, Y \\ $\mathrm{Gu}$; (VI) Manuscript writing: All authors; (VII) Final approval of manuscript: All authors. \\ \#These authors contributed equally to this work. \\ Correspondence to: Zhen Hu. Department of Breast Surgery, Key Laboratory of Breast Cancer in Shanghai, Fudan University Shanghai Cancer Center, \\ Department of Oncology, Shanghai Medical College, Fudan University, Shanghai, China. Email: zhenhu@fudan.edu.cn; Yajia Gu. Department \\ of Radiology, Fudan University Cancer Center, Department of Oncology, Shanghai Medical College, Fudan University, Shanghai, China. \\ Email: cjr.guyajia@vip.163.com.
}

Background: To determine the histopathological and MRI features of BRCA1/2 mutation-associated familial breast cancers compared with those of BRCA1/2 mutation-negative and sporadic breast cancers and to further compare the imaging features of cancers from BRCA1 and BRCA2 mutation carriers according to lesion type on MRI.

Methods: A retrospective review of medical records was conducted to determine tumour clinicopathologic features and MRI characteristics between June 2011 and July 2017, and 93 lesions with BRCA mutations, 93 lesions without BRCA mutations from familial breast cancers and 93 lesions from sporadic breast cancers were included. Histopathologic data, including immunohistochemistry findings and MRI data according to the BI-RADS lexicon, were reviewed. The association between MRI or histopathologic findings and BRCA mutations was analysed.

Results: BRCA-positive familial breast cancers had a higher number of IDCs with high nuclear grade and lymph node metastasis (all $\mathrm{P}<0.05$ ), while the BRCA-negative group had a significantly lower Ki-67 index $(\mathrm{P}<0.001)$. BPE on MRI was found to be significantly lower for BRCA mutations of familial breast cancer $(\mathrm{P}=0.024)$. BRCA1 carriers tended to exhibit the triple-negative phenotype with a more benign shape and margin ( $\mathrm{P}=0.006$ and 0.019$)$, whereas $\mathrm{BRCA} 2$ mutations were associated with the luminal phenotype and more malignant features.

Conclusions: BRCA mutation carriers had a significantly higher number of IDCs with more aggressive cancer, and BRCA-negative cancers had low proliferation levels. Background features on MRI may help to identify BRCA status, while tumour characteristics can differentiate the BRCA1/2 mutation status, consistent with the differences in their clinicopathologic features.

Keywords: Breast cancer; BRCA mutation; magnetic resonance imaging; familial breast cancer

Submitted May 06, 2020. Accepted for publication Nov 27, 2020.

doi: $10.21037 /$ gs-20-596

View this article at: http://dx.doi.org/10.21037/gs-20-596 


\section{Introduction}

BRCA1/2 mutation carriers have an increased risk of developing breast cancer (1). It has been reported that the cumulative risk for developing breast cancer by the age of 70 years ranges from $49 \%$ to $57 \%$ among BRCA $1 / 2$ mutation carriers (2) The BRCA1 and BRCA2 genes are involved in DNA repair and recombination, are located on chromosomes 13 and 17, respectively, and show dominant inheritance (3). Therefore, BRCA1/2 mutation-associated breast cancer has obvious familial aggregation.

Studies have shown that the clinical and pathological features of BRCA1/2 mutation-related breast cancers in familial breast cancers are distinct from those of sporadic breast cancers (4). BRCA1 mutation-containing cancers are more likely to be triple-negative breast cancers with high nuclear grade than sporadic breast cancers (5). In contrast, BRCA2-associated cancers are more commonly found to be ER-positive, and their pathologic characteristics are more similar to those of sporadic cancers (6). The strategies of BRCA-related breast cancer treatment should differ from those for sporadic cancers. It has been suggested that breast cancer patients with BRCA1/2 mutations may benefit from precision treatments, such as platinum-based chemotherapy and poly (ADP-ribose) polymerase inhibitors. These data highlight the need to determine BRCA status in breast cancers (7). Thus far, several studies have focused on the assessment of BRCA1/2 mutation carriers in familial breast cancer (8).

According to the NCCN guideline, regular selfexamination and physical examination can be used to monitor breast cancer occurrence in female BRCA1/2 mutation carriers. In addition, annual screening with contrast-enhanced breast MRI and mammogram with tomosynthesis is also strongly recommended for these women. Multiple studies have demonstrated that the sensitivity of magnetic resonance imaging (MRI) is significantly higher than that of mammography for detecting breast cancer in high-risk women (9-11). MRI has been recommended as the supplemental screening modality for high-risk patients, including women with a known BRCA1/2 mutation and those with a lifetime risk of $20 \%$ or greater for developing breast cancer (12). Data from several studies suggested that MRI features differ according to BRCA mutation type and reflect intrinsic hereditary characteristics. Breast cancer with BRCA1 mutations tends to present as triple-negative cancer and with benign morphologic features but more aggressive tumours $(7,11)$. In contrast, BRCA2-related cancers are more likely to present as ductal carcinoma in situ (DCIS) or ductal carcinoma with the luminal B subtype $(7,9,10)$. To our knowledge, even a few studies have been conducted to examine the relationships between BRCA1/2 mutations and MRI features, there are lack of data in Chinese population. Moreover, none of previous studies investigated whether there was any difference between BRCA mutation positive and negative carriers in familial breast cancers.

Therefore, we aimed to investigate the population of BRCA mutation carriers among familial breast cancers and selected two groups to serve as controls, including one group of BRCA mutation-negative patients with a family history of familial breast cancer and one group of patients with sporadic breast cancer without a family history. The purpose of this study was to determine the histopathologic and MRI features of BRCA1/2 mutation-associated familial breast cancers in a Chinese population and compare them to those of individuals without a BRCA1/2 mutation or with sporadic breast cancer and then to further compare imaging features between cancers with BRCA1 and BRCA2 mutations according to lesion type on MRI. We present the following article in accordance with the MDAR reporting checklist (available at http://dx.doi. org/10.21037/gs-20-596).

\section{Methods}

\section{Patient population}

The study was conducted in accordance with the Declaration of Helsinki (as revised in 2013). This retrospective study was approved by the Medical Ethics Committee of Fudan University Shanghai Cancer Center (No. 1412142-42), and written informed consent was obtained from all patients. All patients with BRCA mutations examined in our hospital between June 2011 and July 2017 were considered. The inclusion criteria were (I) patients with familial breast cancer; (II) patients who underwent genetic testing for BRCA mutations using nextgeneration sequencing technology; and (III) patients who underwent MRI examination before biopsy and previous treatment. Familial breast cancer was defined as previously reported: (I) age younger than or equal to 35 years with at least one other blood relative suffering from any type of cancer; (II) age older than 35 and younger than or equal to 50 years with 2 blood relatives in the same lineage suffering from any type of cancer; or (III) age older than 50 years 
with 3 blood relatives in the same lineage suffering from any type of cancer. The genetic testing technology, as well as the screening and validation of mutated genes, have been described in detail in our previous study (13).

Finally, 87 patients with BRCA mutations and 6 patients with bilateral breast cancers were enrolled in our study. In total, 93 lesions had BRCA mutations, including 50 lesions with BRCA1 and 43 lesions with BRCA2 mutations. Additionally, a group of BRCA mutation-negative patients selected from families with familial breast cancers and a group of sporadic breast cancer cases without a family history served as controls and were matched to the cases in the BRCA mutation group. Ultimately, 93 lesions that were negative for BRCA mutations and 93 cases with sporadic breast cancer were enrolled in this study.

\section{Imaging techniques}

MRI examinations were performed on an Aurora 1.5-T Dedicated Breast MRI system (Aurora Imaging Technology, Inc., Canada) or GE 3.0T system (Signa HDxt; GE Healthcare, Milwaukee, WI, USA) with a dedicated breast coil. On the Aurora system, images were acquired with routine T1W gradient echo sequences (TR/TE 12.9/5.3 ms; thickness $5.0 \mathrm{~mm}$ ) and $\mathrm{T} 2 \mathrm{~W}$ fat-suppressed fast spin echo (FSE) sequences (TR/TE 6,680/29 ms; thickness $5.0 \mathrm{~mm}$ ) before contrast injection. Dynamic imaging was performed with the T1W fat-suppressed sequence (TR/TE 29/4.8 ms; thickness $1.5 \mathrm{~mm}$ ). Contrast medium (Magnevist $0.1 \mathrm{mmol} / \mathrm{kg}$; Bayer Schering Pharma AG, Berlin, Germany) was administered as a bolus injection followed by a 20-mL saline flush. Images were obtained at 90, 180, 270 and 360 seconds after injection. On the GE system, a T1 FSE sequence (TR/TE 8,600/10.5 ms; thickness $2.0 \mathrm{~mm}$ ) and a short time inversion recovery (STIR) sequence (TR/ TE 3,600/100 ms; thickness $2.0 \mathrm{~mm}$ ) were performed before contrast. Contrast agents (Magnevist; Bayer Schering Pharma, Berlin, Germany) were injected $(0.2 \mathrm{mmol} / \mathrm{kg}$; flow rate $2 \mathrm{~ms} / \mathrm{s}$ ) followed by a $20-\mathrm{mL}$ saline flush. A series of seven $\mathrm{T} 1 \mathrm{~W}$ fat-suppressed enhanced images were obtained with a Vibrant sequence (TR/TE $6.5 / 3.5 \mathrm{~ms}$; thickness $3 \mathrm{~mm})$. The Hospital Information System and the Picture Archiving and Communication System were available to radiologists for the interpretation of images.

\section{Pathologic characteristics}

All pathologic results were defined according to the World
Health Organization classification of breast tumours (14), including tumour type (including DCIS, invasive ductal carcinoma (IDC) and other types), tumour size, the presence of lymphovascular invasion, the presence of lymph node metastasis, and $\mathrm{Ki}-67$ positivity.

According to IHC and tumour proliferation based on the St Gallen consensus (15), breast cancers were divided into three molecular types: (I) the luminal subtype (ER or PR positive (or both), HER2 negative or positive); (II) the HER2 subtype, non-luminal (HER2 positive and ER and PR negative); or (III) the triple-negative subtype (HER2 negative and $\mathrm{ER}$ and $\mathrm{PR}$ negative).

\section{Imaging review}

The MR features of the breast cancer patients were analysed using the American College of Radiology Breast Imaging Reporting and Data System (BI-RADS) lexicon (16) and included fibroglandular tissue (FGT), breast parenchymal enhancement (BPE), and morphologic features including mass, non-mass enhancement (NME) or both; internal enhancement, solitary or multiple; the kinetic curve; and others. BI-RADS categories were also recorded. All MR images were reviewed by 2 breast radiologists (with 10 and 16 years of experience) who were blinded to the mutation status and medical history of each patient. If there was disagreement, a radiologist with more seniority was invited to form a final conclusion.

\section{Statistical analysis}

The data were analysed using SPSS 16.0 software (SPSS Inc., Chicago, IL, USA). The Pearson chi-squared test or Fisher's exact test was used to compare the clinical and MR characteristics across the three patient groups (BRCA mutation group, BRCA-negative group, and sporadic breast cancer group), as well as between the BRCA1 and BRCA2 mutation groups. The Kruskal-Wallis exact test was used to compare the median ages of the different groups. $\mathrm{P}<0.05$ was considered statistically significant.

\section{Results}

\section{Patient cohort and tumour characteristics}

Table 1 summarizes the clinical and pathologic characteristics of all the cases. All cases were evenly divided into the BRCA mutation-positive familial breast cancer group, 
Table 1 The clinicopathological characteristics of all the cases

\begin{tabular}{|c|c|c|c|c|c|c|}
\hline Characteristics & \multicolumn{2}{|c|}{$\mathrm{BRCA}+(\mathrm{n}=93), \mathrm{n}(\%)$} & $\begin{array}{c}\text { BRCA }-(n=93), \\
n(\%)\end{array}$ & $\begin{array}{c}\text { Sporadic }(n=93) \\
n(\%)\end{array}$ & $\mathrm{P}_{1}$ & $\mathrm{P}_{2}$ \\
\hline Age, years & $41.28 \pm 8.48$ & $42.86 \pm 9.44$ & $41.66 \pm 11.53$ & $42.16 \pm 10.78$ & 1.27 & 0.39 \\
\hline Menopausal status & & & & & 0.37 & 0.38 \\
\hline Pre- & $43(86)$ & $34(79.07)$ & $68(73.12)$ & $72(77.42)$ & & \\
\hline Histology & & & & & $0.04^{*}$ & 0.08 \\
\hline IDC & $46(92)$ & $39(90.7)$ & $72(77.42)$ & $81(87.1)$ & & \\
\hline DCIS & $1(2)$ & $4(9.3)$ & $10(10.75)$ & $9(9.68)$ & & \\
\hline Others & $3(6)$ & $0(0.0)$ & $11(11.83)$ & $3(3.22)$ & & \\
\hline 2 & $22(44)$ & $21(48.84)$ & $40(43.01)$ & $52(55.91)$ & & \\
\hline 3 & $3(6)$ & $1(2.32)$ & $6(6.45)$ & $5(5.38)$ & & \\
\hline Lymph node & & & & & $0.04^{*}$ & 0.09 \\
\hline Negative & $32(64)$ & $20(46.51)$ & $59(63.44)$ & $67(72.04)$ & & \\
\hline Positive & $18(36)$ & $23(54.49)$ & $34(36.56)$ & $26(27.96)$ & & \\
\hline Lymphovascular invasion & & & & & 0.66 & 0.54 \\
\hline Negative & $34(68)$ & $26(60.47)$ & $66(70.97)$ & $61(65.59)$ & & \\
\hline Ki-67 & & & & & $<0.001^{*}$ & 0.36 \\
\hline$\leq 14$ & $7(14)$ & $8(18.6)$ & $38(40.86)$ & $11(11.83)$ & & \\
\hline$>14$ & $43(86)$ & $35(81.4)$ & $55(59.14)$ & $82(88.17)$ & & \\
\hline Nuclear grade of IDC & & & & & $<0.001^{*}$ & 0.16 \\
\hline 1 & $0(0.0)$ & $2(5.13)$ & $1(1.39)$ & $0(0.0)$ & & \\
\hline 2 & $10(21.74)$ & $12(30.77)$ & 39 (54.17) & $44(54.32)$ & & \\
\hline 3 & 36 (78.26) & $25(64.1)$ & $32(44.44)$ & 37 (45.68) & & \\
\hline
\end{tabular}

Numerical data are presented as the mean \pm SD. Nonnumerical data are resented as the number of patients (percentage). The $P_{1}$ value was compared with the three patient groups (BRCA mutation group, BRCA negative group and sporadic breast cancer group), and the $\mathrm{P}_{2}$ value was compared between BRCA1 and BRCA2 mutation groups. *, $\mathrm{P}<0.05$; $^{\mathrm{a}}$, two cases of malignant lobular tumour without molecular subtypes in BRCA negative group. IDC, invasive ductal carcinoma; DCIS, ductal carcinoma in situ; HER2, human epidermal growth factor 2. 
Table 2 MRI features of the all the cases

\begin{tabular}{|c|c|c|c|c|c|c|}
\hline Features & \multicolumn{2}{|c|}{$\mathrm{BRCA}+(n=93), n(\%)$} & $\begin{array}{c}\text { BRCA- }(n=93), \\
n(\%)\end{array}$ & $\begin{array}{c}\text { Sporadic }(n=93) \\
n(\%)\end{array}$ & $\mathrm{P}_{1}$ & $\mathrm{P}_{2}$ \\
\hline FGT & & & & & 0.13 & 0.38 \\
\hline $1+2$ & $3(6)$ & $1(2.33)$ & $3(3.23)$ & $9(9.68)$ & & \\
\hline $3+4$ & 47 (94) & $42(97.67)$ & $90(96.77)$ & $84(90.32)$ & & \\
\hline $1+2$ & $39(78)$ & 31 (72.09) & 57 (61.29) & $53(56.99)$ & & \\
\hline $3+4$ & $11(22)$ & $12(27.91)$ & $36(41.94)$ & $40(43.01)$ & & \\
\hline Lesion type & & & & & 0.053 & $0.03^{*}$ \\
\hline Mass & $46(92)$ & 31 (72.09) & $74(79.57)$ & $68(73.12)$ & & \\
\hline $\mathrm{T} 2 \mathrm{WI}$ & & & & & 0.19 & 0.11 \\
\hline Hypointensity & $16(32)$ & $11(25.58)$ & $32(34.41)$ & $20(21.50)$ & & \\
\hline Isointensity & $30(60)$ & $32(74.42)$ & $59(63.44)$ & $72(77.42)$ & & \\
\hline Hyperintensity & $4(8)$ & $0(0.0)$ & $2(2.15)$ & $1(1.08)$ & & \\
\hline Curve type & & & & & $0.038^{*}$ & 0.33 \\
\hline Type I & $2(4)$ & $5(11.63)$ & $6(6.45)$ & $0(0.0)$ & & \\
\hline Type II & $15(30)$ & $14(32.56)$ & 41(44.09) & 39 (41.94) & & \\
\hline Type III & $33(66)$ & $24(55.81)$ & $46(49.46)$ & $54(58.06)$ & & \\
\hline
\end{tabular}

*, $\mathrm{P}<0.05$. FGT was categorized into four levels: 1 = almost entirely fat, 2 = scattered fibroglandular density, $3=$ heterogeneously dense, 4 = extremely dense. BPE was categorized into four levels: $1=$ minimal, 2 = mild, 3 = moderate, $4=$ marked. FGT, fibroglandular tissue; $\mathrm{BPE}$, breast parenchymal enhancement; NME, non-mass enhancement.

BRCA-negative familial breast cancer group and sporadic breast cancer group, each with 93 cases $(33.33 \%, 93 / 279)$. Of the cases in the BRCA mutation group, $50(53.76 \%$, 50/93) had a BRCA1 mutation, $43(46.23 \%, 43 / 93)$ had a BRCA2 mutation, and none had mutations in both BRCA1 and BRCA2. No significant difference in patient age or menopausal status was observed.

When the BRCA-positive, BRCA-negative, and sporadic breast cancer groups were compared, the BRCA-positive group had a significantly higher number of IDCs $(\mathrm{P}=0.04)$ with high nuclear grade $(\mathrm{P}<0.001)$, and most $\mathrm{BRCA}$ mutation carriers had lymph node involvement $(\mathrm{P}=0.04)$. Regarding immunohistochemistry, the distributions of the tumour molecular subtypes and Ki-67 positivity were found to differ significantly according to BRCA mutation status (all $\mathrm{P}<0.001)$. The BRCA-positive group had a significantly higher number of triple-negative breast cancers, and the
BRCA-negative group had a significantly lower Ki-67 index. No significant differences in $\mathrm{T}$ stage or lymphovascular invasion were observed among the three groups.

In the comparison of the BRCA1 and BRCA2 mutationcontaining tumours, those with BRCA1 mutations were associated with the triple-negative subtype (41/50, $82 \%$ ), and those with BRCA2 mutations were associated with the luminal subtype $(31 / 43,72.09 \%)(\mathrm{P}<0.001)$. There were no significant differences in the other clinicopathological characteristics between the BRCA1 and BRCA2 groups.

\section{Associations between imaging features and BRCA mutation status}

All the lesions showed positive findings on MRI (Table 2). Regarding the background features, BPE was found to be significantly lower in both BRCA mutation subgroups 

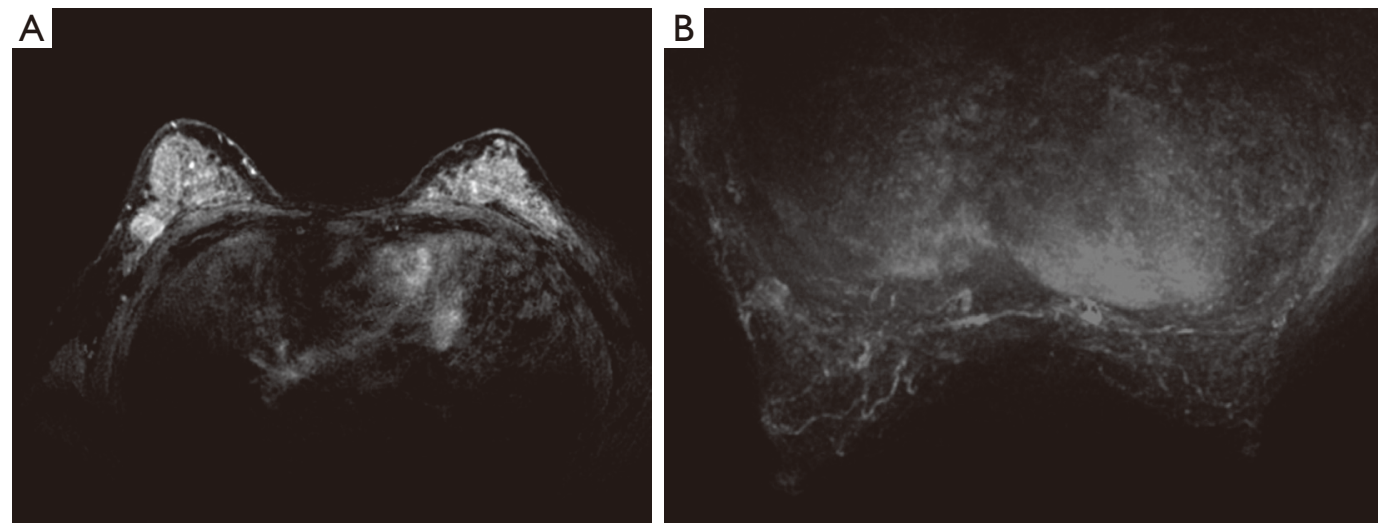

Figure 1 A 44-year-old woman with BRCA1 mutation familial breast cancer and high-grade invasive ductal carcinoma (triple negative subtype). The MRI obtained in the early post-contrast phase (A) and acquired MIP (B) showed a round circumscribed mass with a mild enhancement in the upper outer quadrant of the right breast and the BPE was mild in both breasts. MIP, maximal intensity projection; BPE, background parenchymal enhancement.
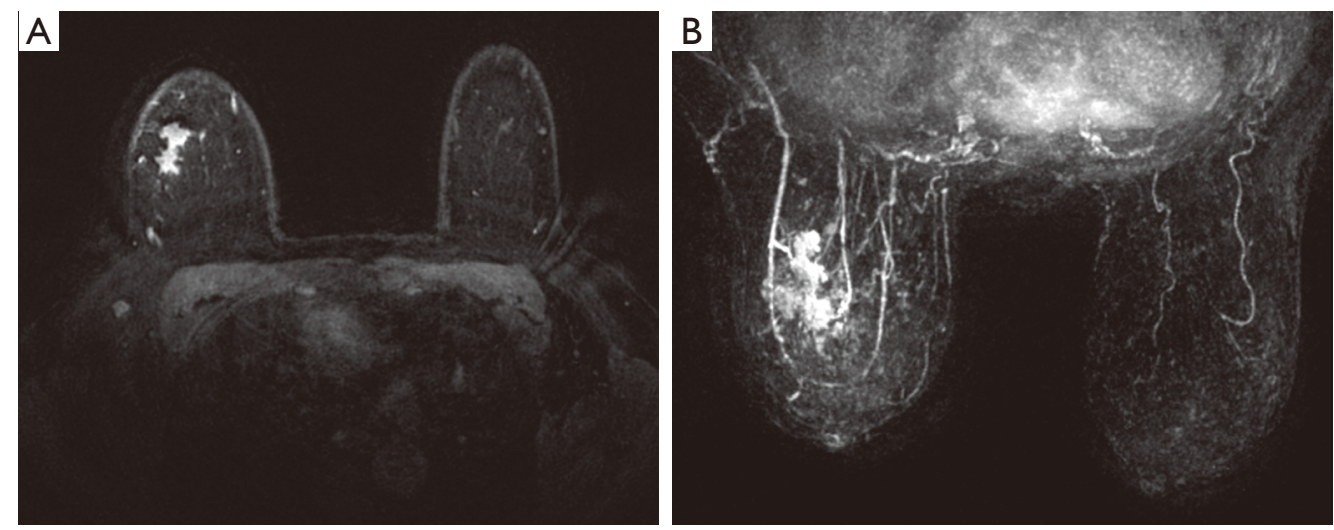

Figure 2 A 43-year-old woman with BRCA2 mutation familial breast cancer and high-grade invasive ductal carcinoma (Luminal subtype). The MRI obtained in the early post-contrast phase (A) and acquired MIP (B) showed an irregular mass with peripheral focal NME in the upper outer quadrant of the right breast and the BPE was minimal in both breasts. MIP, maximal intensity projection; BPE, background parenchymal enhancement; NME, non-mass enhancement.

than in both the BRCA-negative group of familial breast cancer and the sporadic breast cancer group $(\mathrm{P}=0.024)$ (Figures 1,2,3); however, there was no significant difference in FGT. Regarding tumour characteristics, most cancers in the three groups showed a type III enhancement curve with a washout pattern, but the sporadic breast cancer group was found to have no type I enhancement $(\mathrm{P}=0.038)$. BRCA1 carriers tended to show more mass lesions than BRCA2 mutation carriers $(\mathrm{P}=0.017)$. There were no differences in T2WI signal intensity between groups.

\section{Differences in MRI features of the BRCA1 and BRCA2 carriers according to lesion type}

On the basis of the significant difference in lesion type between the BRCA1 and BRCA2 mutation groups, the MR features according to lesion type were assessed, as summarized in Table 3. Mass-type lesions were identified in $92 \%$ and $76.74 \%$ of BRCA1 and BRCA2 mutation cases, respectively, while NME-type lesions were identified in $8 \%$ and $23.26 \%$ of these patients, respectively.

Regarding mass lesions, the shape and margin were 

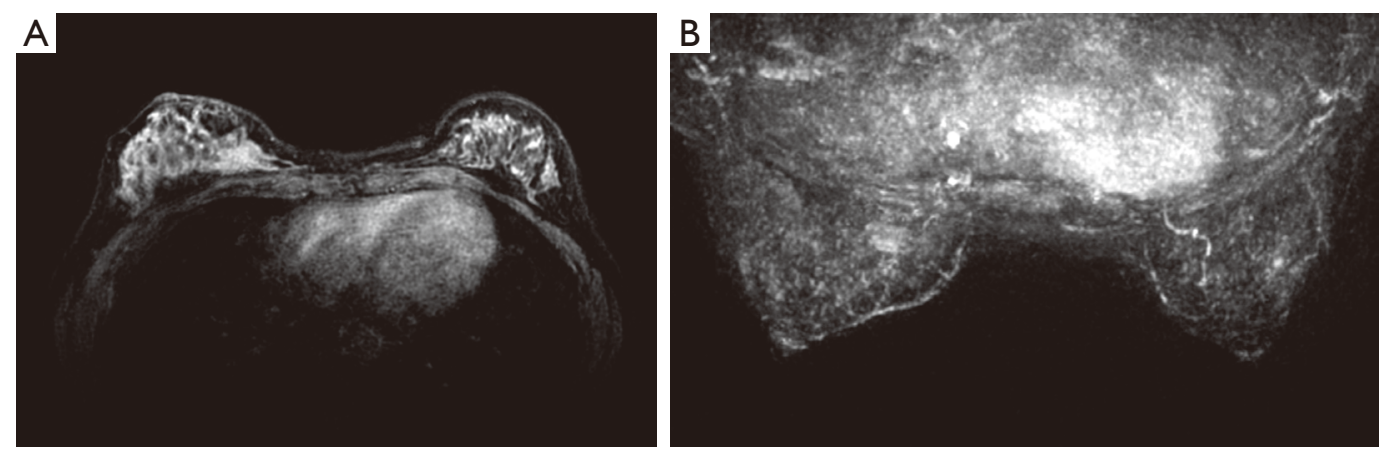

Figure 3 A 38-year-old woman with BRCA-negative familial breast cancer and high-grade invasive ductal carcinoma (Luminal subtype). The MRI obtained in the early post-contrast phase (A) and acquired MIP (B) showed an irregular mass with heterogeneous enhancement in the lower inner quadrant of the right breast and the BPE was moderate in both breasts. MIP, maximal intensity projection; BPE, background parenchymal enhancement.

Table 3 The MRI features of mass-type and NME type lesions between the BRCA1 and BRCA2 mutation groups

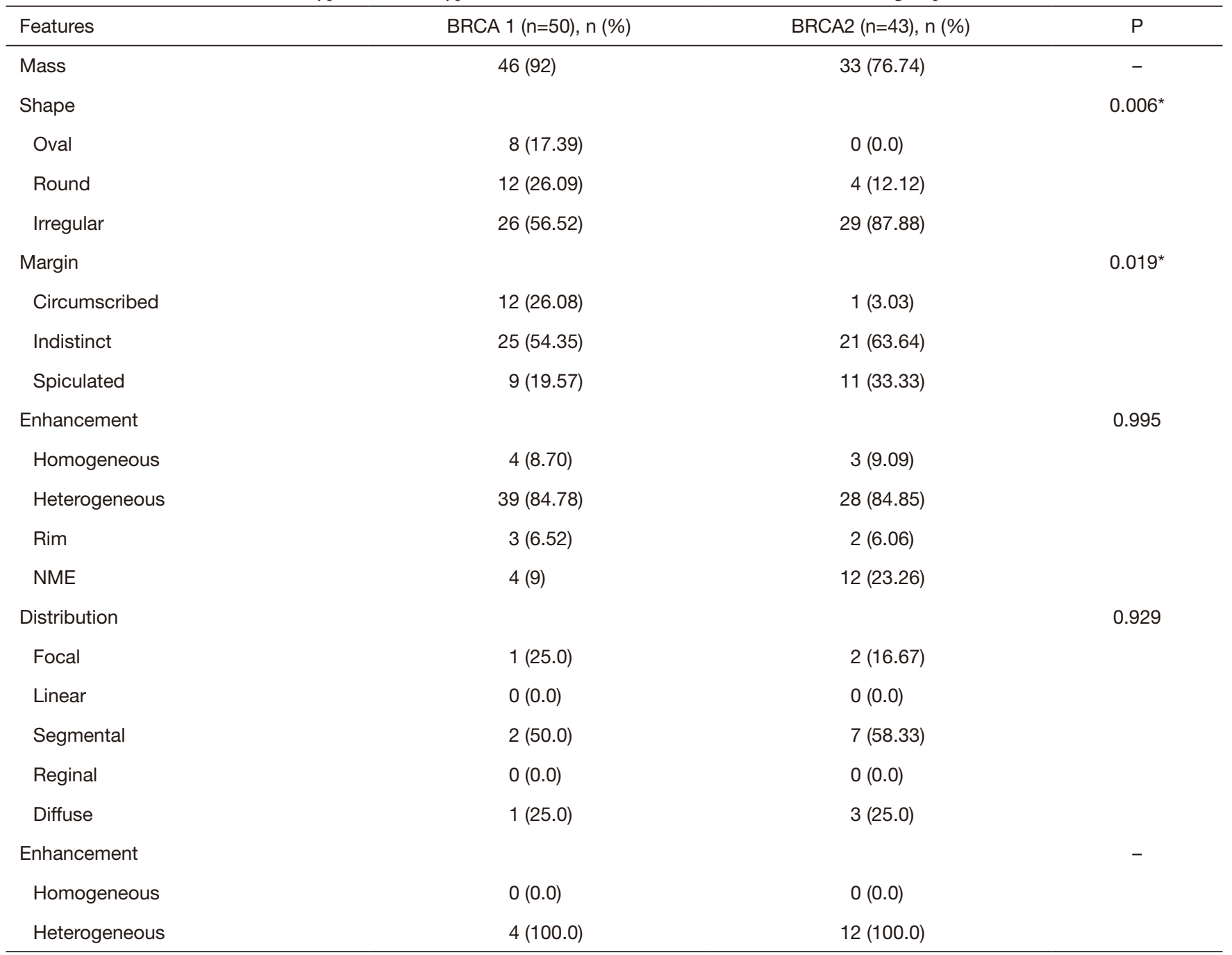

Nonnumerical data are resented as the number of patients (percentage). ${ }^{*}, \mathrm{P}<0.05$. 
found to be significantly different between the BRCA1 and BRCA2 groups ( $\mathrm{P}=0.006$ and 0.019 , respectively). BRCA1 carriers tended to exhibit a more oval or round shape with circumscribed margins than BRCA2 carriers (Figures 1,2). However, the dominant MRI characteristics of both groups included an irregular shape, indistinct margin, and heterogeneous enhancement, which represented typical malignant tumours. Regarding NME lesions, there were no significant differences between the two groups, but segmental distribution and heterogeneous enhancement were the main features in both groups.

\section{Discussion}

In this study, BRCA-positive familial breast cancers had a higher number of IDCs with high nuclear grade and lymph node metastasis by histopathology but had a lower BPE regardless of BRCA1/2 status. The imaging features of BRCA1 carriers differ from those of BRCA2 mutations, consistent with the difference in clinicopathologic features. BRCA-negative cancers had a low Ki-67 index compared with the other two groups, but the MR features of the BRCA-negative cancers were more similar to those of sporadic breast cancers.

Our study included both BRCA-negative carriers with a history familial breast cancer and patients with sporadic breast cancer to form two control groups. In contrast to previous studies that included only BRCA-negative groups as controls $(6,7)$, this study broadened the inclusion criteria to establish the sporadic breast cancer group, which was predicted to have a very low BRCA mutation rate. BRCA mutations accounted for approximately $5 \%$ of all breast cancer patients, and none of the sporadic breast cancers had a family history in our study. Thus, the likelihood of possible mutations was less than $3 \%$, which has been reported in the previous literature (17). The reasons for setting up these two groups were as follows: first, the BRCA-negative mutation group cannot rule out the presence of other genetic mutations or other types of inherited breast cancer, which are completely different from general breast cancer, and second, sporadic breast cancers can reflect the imaging features of general breast cancers.

Interestingly, our results showed that BRCA mutation carriers had a significantly lower BPE than women with BRCA-negative or sporadic breast cancers, while there was no difference in BPE between the BRCA1 and BRCA2 groups. To date, few studies have focused on the background features of MRI in the association between BRCA mutation status and imaging features. Most previous studies suggest that an increased level of BPE can serve as an important risk factor and biomarker for breast cancer and that high levels of BPE may be an indicator in high-risk women (18-20). In contrast, our study suggests that lower BPE correlates with a BRCA-positive mutation status regardless of whether the mutation is in BRCA1 or 2.

The opposite results may be due to differences in the effects of hormones in patients with and without BRCA mutations. The possible reason could be that the instability of $B R C A$ gene mutations, which have special biological significance, may cause tissue remodelling by changing hormone signal transduction, thus affecting the response of decreased BPE to breast epithelial blood supply (21). One hypothesis is that mutations in the BRCA gene and its associated sites activate the hormone receptor negative subtype-specific pathway, and the oncogene mediates DNA replication stress, thus directly triggering and promoting tumour progression (22). In this study, the BRCA-positive group, especially the BRCA1 mutation carrier group, had a significantly higher number of triple-negative breast cancers, which is in line with previous literatures $(4,6,7,23)$. The absence of oestrogen receptors and progesterone receptors may lead to a decrease in BPE in BRCA mutation carriers. However, there was no difference in BPE between BRCA 1 and BRCA2 carriers. Since BRCA2 tumours are associated with the luminal subtype that usually exhibits ER positivity, the lower BPE in BRCA2 mutation carriers suggests that the $B R C A 2$ gene has multifactorial and complex biologic implications and still needs further study.

In agreement with previous observations, BRCA1 mutation-containing breast cancers more frequently manifested as benign lesions with round or oval shapes, circumscribed margins, and rim enhancement, while fibroadenoma-like masses were found on MRI scans in $23 \%$ of invasive cancers in women with familial risk $(7,10,24)$. Because BRCA1 mutations are associated with a triple-negative phenotype and aggressive pathological characteristics, the features identified by MRI in the tumours of patients with BRCA1 mutations may match those described above. Regarding mass lesions, BRCA1 carriers tended to exhibit a more oval or round shape with circumscribed margins in our study. Most BRCA2 cancers had irregular shapes, indistinct or spiculate margins, and heterogeneous enhancement on MRI, findings that were consistent with previous findings (25).

The other significant finding of tumour features was the enhancement curve type in our study. Even though most 
cancers in each group showed type II and III enhancement curves, there was a certain percentage of cancer with type I curves; a benign curve was usually apparent in the BRCApositive and BRCA-negative familial breast cancers, while the sporadic breast cancer group was found to have no type I enhancement. A previous study found no significant difference in enhancement kinetics because they included only BRCA-negative breast cancers as a control (7). For this result, it was still necessary to enlarge the sample size for further exploration.

In addition to focusing on BRCA mutations, our study also sought to investigate the characteristics of BRCAnegative familial breast cancer. BRCA-negative cancers had a significantly lower Ki-67 index than the BRCA-positive and sporadic breast cancers. A previous study demonstrated that low expression of the $\mathrm{Ki}-67$ antigen, which is used to evaluate proliferative activity in a breast cancer, indicates a low risk of relapse and a better survival probability (26). The prognostic significance of the Ki-67 scoring categories in breast cancer subgroups has been shown. However, the MR features of BRCA-negative cancers were more similar to those of sporadic breast cancers.

There are several limitations of this study. First, the number of patients in each group was relatively small, and our analysis was retrospective. Second, we have only recently begun to evaluate the MRI characteristics of these breast cancers. The other imaging systems including mammography and ultrasonography as well as integrated interpretation are still essential. Third, further studies with follow-up and prognostic information need to be conducted in order to elucidate the relationship between the genetic and molecular subtypes. Fourth, this study was performed in a retrospective diagnostic population, so future studies may need a broader prospective screening setting.

\section{Conclusions}

In summary, our study suggests that BPE on MRI is significantly lower in BRCA mutation carriers with familial breast cancer and that BRCA mutation carriers also have a significantly higher number of IDCs with high nuclear grade and lymph node metastasis. BRCA1 mutation carriers tend to exhibit the triple-negative phenotype with more benign features, whereas BRCA2 mutations are associated with the luminal phenotype and present more malignant features. The background features on MRI may help to identify BRCA status, while tumour characteristics could differentiate the BRCA1/2 mutation status. BRCA-negative cancers have a low $\mathrm{Ki}-67$ index compared with the other two groups, while the MR features of BRCA-negative cancers are not different from those of sporadic breast cancers. To elucidate the genetic differences, further studies integrating imaging findings and prognostic information are still needed.

\section{Acknowledgments}

We thank all the patients of this study for their participation. We greatly appreciate Yaosheng Wang, Lei Yue and Min Qian at Department of Radiology for their excellent assistance. We thank AJE for the language editing.

Funding: This project was supported by the grants from The National Natural Science Foundation of China (81901703, 82071878), Shanghai Municipal Health Planning Commission Youth Project (20184Y0010), Youth Medical Talents-Clinical Imaging Practitioner Program [SHWRS(2020)_087], and Clinical Research Plan of SHDC (SHDC2020CR2008A).

\section{Footnote}

Reporting Checklist: The authors have completed the MDAR reporting checklist. Available at http://dx.doi.org/10.21037/ gs-20-596

Data Sharing Statement: Available at http://dx.doi. org/10.21037/gs-20-596

Conflicts of Interest: All authors have completed the ICMJE uniform disclosure form (available at http://dx.doi. org/10.21037/gs-20-596). The authors have no conflicts of interest to declare.

Ethical Statement: The authors are accountable for all aspects of the work in ensuring that questions related to the accuracy or integrity of any part of the work are appropriately investigated and resolved. The study was approved by the Medical Ethics Committee of Fudan University Shanghai Cancer Center, Shanghai, China (approval number 1412142-42), and written informed consent was obtained from all patients. The study was conducted in accordance with the Declaration of Helsinki (as revised in 2013).

Open Access Statement: This is an Open Access article distributed in accordance with the Creative Commons 
Attribution-NonCommercial-NoDerivs 4.0 International License (CC BY-NC-ND 4.0), which permits the noncommercial replication and distribution of the article with the strict proviso that no changes or edits are made and the original work is properly cited (including links to both the formal publication through the relevant DOI and the license). See: https://creativecommons.org/licenses/by-nc-nd/4.0/.

\section{References}

1. Krainer M, Silva-Arrieta S, FitzGerald MG, et al. Differential contributions of BRCA1 and BRCA2 to earlyonset breast cancer. N Engl J Med 1997;336:1416-21.

2. Chen S, Parmigiani G. Meta-analysis of BRCA1 and BRCA2 penetrance. J Clin Oncol 2007;25:1329-33.

3. Venkitaraman AR. Cancer susceptibility and the functions of BRCA1 and BRCA2. Cell 2002;108:171-82.

4. Atchley DP, Albarracin CT, Lopez A, et al. Clinical and pathologic characteristics of patients with BRCApositive and BRCA-negative breast cancer. J Clin Oncol 2008;26:4282-8.

5. Rakha EA, Reis-Filho JS, Ellis IO. Basal-like breast cancer: a critical review. J Clin Oncol 2008;26:2568-81.

6. Noh JM, Han BK, Choi DH, et al. Association between BRCA Mutation Status, Pathological Findings, and Magnetic Resonance Imaging Features in Patients with Breast Cancer at Risk for the Mutation. J Breast Cancer 2013;16:308-14.

7. Ha SM, Chae EY, Cha JH, et al. Association of BRCA Mutation Types, Imaging Features, and Pathologic Findings in Patients With Breast Cancer With BRCA1 and BRCA2 Mutations. AJR Am J Roentgenol 2017;209:920-8.

8. Schrading S, Kuhl CK. Mammographic, US, and MR imaging phenotypes of familial breast cancer. Radiology 2008;246:58-70.

9. Krammer J, Pinker-Domenig K, Robson ME, et al. Breast cancer detection and tumor characteristics in BRCA1 and BRCA2 mutation carriers. Breast Cancer Res Treat 2017;163:565-71.

10. Kuhl C, Weigel S, Schrading S, et al. Prospective multicenter cohort study to refine management recommendations for women at elevated familial risk of breast cancer: the EVA trial. J Clin Oncol 2010;28:1450-7.

11. Sung JS, Stamler S, Brooks J, et al. Breast Cancers Detected at Screening MR Imaging and Mammography in Patients at High Risk: Method of Detection Reflects Tumor Histopathologic Results. Radiology
2016;280:716-22.

12. Bevers TB, Helvie M, Bonaccio E, et al. Breast Cancer Screening and Diagnosis, Version 3.2018, NCCN Clinical Practice Guidelines in Oncology. J Natl Compr Canc Netw 2018;16:1362-89.

13. Yang $\mathrm{X}, \mathrm{Wu} \mathrm{J}, \mathrm{Lu} \mathrm{J}$, et al. Identification of a Comprehensive Spectrum of Genetic Factors for Hereditary Breast Cancer in a Chinese Population by Next-Generation Sequencing. PLoS One 2015;10:e 0125571.

14. Lebeau A, Kriegsmann M, Burandt E, et al. Invasive breast cancer: the current WHO classification. Pathologe 2014;35:7-17.

15. Goldhirsch A, Winer EP, Coates AS, et al. Personalizing the treatment of women with early breast cancer: highlights of the St Gallen International Expert Consensus on the Primary Therapy of Early Breast Cancer 2013. Ann Oncol 2013;24:2206-23.

16. Mercado CL. BI-RADS update. Radiol Clin North Am 2014;52:481-7.

17. Apostolou P, Fostira F. Hereditary breast cancer: the era of new susceptibility genes. Biomed Res Int 2013;2013:747318.

18. King V, Brooks JD, Bernstein JL, et al. Background parenchymal enhancement at breast MR imaging and breast cancer risk. Radiology 2011;260:50-60.

19. Dontchos BN, Rahbar H, Partridge SC, et al. Are Qualitative Assessments of Background Parenchymal Enhancement, Amount of Fibroglandular Tissue on MR Images, and Mammographic Density Associated with Breast Cancer Risk? Radiology 2015;276:371-80.

20. Chen JH, Yu HJ, Hsu C, et al, Background Parenchymal Enhancement of the Contralateral Normal Breast: association with Tumor Response in Breast Cancer Patients Receiving Neoadjuvant Chemotherapy. Transl Oncol 2015;8:204-9.

21. Stevens KN, Vachon CM, Couch FJ. Genetic susceptibility to triple-negative breast cancer. Cancer Res 2013;73:2025-30.

22. Curtis C, Shah SP, Chin SF, et al. The genomic and transcriptomic architecture of 2,000 breast tumours reveals novel subgroups. Nature 2012;486:346-52.

23. You C, Kaiser AK, Baltzer P, et al. The assessment of background parenchymal enhancement (BPE) in a high-risk population: What causes BPE? Transl Oncol 2018;11:243-9.

24. Uematsu T, Kasami M, Yuen S. Triple-negative breast 
cancer: correlation between MR imaging and pathologic findings. Radiology 2009;250:638-47.

25. Gilbert FJ, Warren RM, Kwan-Lim G, et al. Cancers in BRCA1 and BRCA2 carriers and in women at high risk for breast cancer: MR imaging and mammographic features.

Cite this article as: You C, Xiao Q, Zhu X, Sun Y, Di G, Liu G, Hou Y, Chen C, Wu J, Shao Z, Gu Y, Hu Z. The clinicopathological and MRI features of patients with BRCA1/2 mutations in familial breast cancer. Gland Surg 2021;10(1):262272. doi: 10.21037 /gs-20-596
Radiology 2009;252:358-68.

26. Niikura N, Masuda S, Kumaki N, et al. Prognostic significance of the $\mathrm{Ki}-67$ scoring categories in breast cancer subgroups. Clin Breast Cancer 2014;14:323-9.e3. 